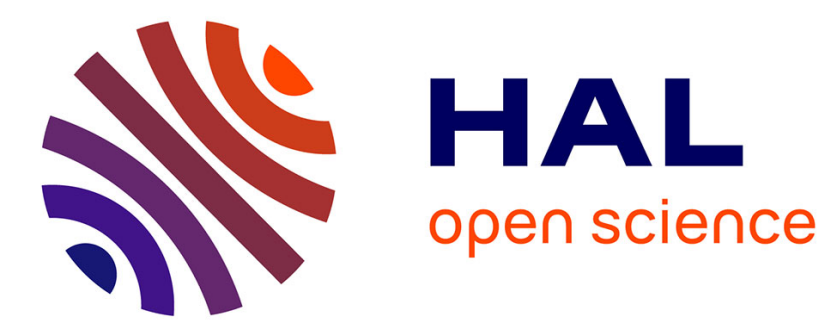

\title{
Terahertz Differential Computed Tomography: a Relevant Nondestructive Inspection Application
}

\author{
Alexandre Duhant, Meriam Triki, Olivier Strauss
}

\section{To cite this version:}

Alexandre Duhant, Meriam Triki, Olivier Strauss. Terahertz Differential Computed Tomography: a Relevant Nondestructive Inspection Application. Journal of Infrared, Millimeter and Terahertz Waves, 2019, 40 (2), pp.178-199. 10.1007/s10762-018-0564-5 . hal-01968434

\section{HAL Id: hal-01968434 https://hal.science/hal-01968434}

Submitted on 3 Jan 2019

HAL is a multi-disciplinary open access archive for the deposit and dissemination of scientific research documents, whether they are published or not. The documents may come from teaching and research institutions in France or abroad, or from public or private research centers.
L'archive ouverte pluridisciplinaire HAL, est destinée au dépôt et à la diffusion de documents scientifiques de niveau recherche, publiés ou non, émanant des établissements d'enseignement et de recherche français ou étrangers, des laboratoires publics ou privés. 


\title{
Terahertz differential computed tomography: a relevant non destructive inspection application
}

\author{
Alexandre Duhant · Meriam Triki · Olivier Strauss
}

Received: date / Accepted: date

\begin{abstract}
In recent years, tremendous advances have been made in the choice of materials used in industry. With weight reduction as the goal, composite and polymer materials are more and more popular but they are almost transparent to X-ray. Because of this, interest has grown in other wavelengths like TeraHertz $(\mathrm{THz})$. Due to a difference in how X-ray and $\mathrm{THz}$ propagate, X-ray CT algorithms cannot be directly used. For example, THz induces refraction making the reconstruction problem nonlinear. In this paper, we present a new algorithm which complies with beam profile intensities, refraction, reflection. It is based on linearizing the reconstruction process around a Computer Aided Design (CAD) model of the object to be reconstructed. The method we propose computes the deviation between the object and this model.
\end{abstract}

Keywords Terahertz computed tomography $\cdot$ Invers problem $\cdot$ Non destructive testing . Refraction $\cdot$ Non linear problem $\cdot$ Modeling $\cdot$ Projection simulation $\cdot$ Monte Carlo

\section{Introduction}

Today in an industrial context, many efforts have been made to improve the choice and knowledge of manufacturing and control processes for materials and/or the assembly of materials whilst reducing cost and time. As a consequence, study and development of non invasive monitoring and performance solutions are required.

\section{A. Duhant}

LIRMM, University of Montpellier, CNRS, Montpellier, France

Department of Research and Development, T-Waves Technologies, Montpellier, France

E-mail: alexandre.duhant@lirmm.fr

M. Triki

Department of Research and Development, T-Waves Technologies, Montpellier, France

E-mail: meriam.triki@t-waves-technologies.com

O. Strauss

LIRMM, University of Montpellier, CNRS, Montpellier, France

E-mail: olivier.strauss@lirmm.fr 
Tomography is a method to retrieve internal 3D structures of an object based on 2D external measurements. Its usage in Non Destructive Testing (NDT) has become popular since it allows us to inspect three dimensional manufactured objects. Among the three main modalities of tomography (magnetic resonance [16], transmission tomography [11] and emission tomography [2]), only transmission Computed Tomography (CT) is currently used in NDT [3].

Usual transmission CT measurements are obtained by directing X-rays at an object from different orientations and measuring the decreasing intensity with detectors positioned on the opposite side of the X-ray source. CT consists of reconstructing a sampled density map (or more precisely an image of mean absorption coefficients) based on considering how each ray has been attenuated by the object. Such a reconstruction involves solving an inverse problem.

Over the last decade, interest in X-ray CT has grown significantly as a technique for NDT as reviewed by Stock [20]. Although tomography is increasingly present in modern industrial applications, its use is limited by the nature of the available radiation necessary for characterization of the employed materials, since some materials used under industrial context are nearly transparent to X-ray due to their low density. As a last drawback, X-ray has security implications, limiting the size of inspected objects and this results in bulky tomograph.

Tomography based on Terahertz (THz) waves, since they range 0.1 to $10 \mathrm{THz}$, could be a possible solution to the previously mentioned limitations. It does not have security implications as restrictive as X-rays and, due to their relatively long wave length, compared to X-rays, they interact with materials that are transparent to X-rays. However, there is a drawback: inspections made with $\mathrm{THz}$ radiations are of limited spatial resolution. Moreover, $\mathrm{THz}$ waves are completely blocked by water and, as for X-rays, reflected by metals.

After the seminal work proposed in [12], THz CT has become popular due to the emergence of new detectors and sources. However, reconstructing a density map based on a $\mathrm{THz}$ measurements is not straightforward since phenomena like beam steering (i.e. refraction) or reflection loss must be taken into account. The advent of these phenomena highly depends on both the shape and materials of the acquired object. Moreover, THz beam diverges faster than X-rays, which mostly propagate as straight ray. Therefore, the relation between the beam attenuation and the densities to be reconstructed cannot be represented by a linear relation anymore. This should prohibits the direct use of conventional reconstruction algorithms [13].

Even so, some attempts at reconstructing an attenuation map based on $\mathrm{THz}$ measurements by using conventional methods have been proposed in literature, some by hypothesizing that both reflection and refraction are negligible, others by using some prior information on the object under inspection.

In this paper we propose a new approach that is completely relevant to the context of NDT. It consists in supposing that the object under inspection slightly deviates from a known Computer Aided Design (CAD) model, and to reconstruct this deviation instead of directly reconstructing the object. This method is based on linearizing the reconstruction problem around its CAD model. We propose a new way of modeling the interaction between material and $\mathrm{THz}$ waves that makes this reconstruction possible in reasonable computation time and with an acceptable level of accuracy. The method we propose is dedicated to a focused $\mathrm{THz}$ beam. We consider 2D tomography, i.e. reconstructing an object slice by slice. The 3D extension of this process is rather straightforward, but very computationally expensive as it would be for X-ray CT. 


\section{Towards a THz tomography}

\subsection{Classical tomographic reconstruction}

Several algorithms have been used in the long history of CT to deal with inverting the measurement model and obtaining the inner structure of inspected objects [11]. All these algorithms are based on a Radon-like linear modeling of the attenuation the wave undergoes by passing through an object. Let $J$ be the vector of density values of the slice to be reconstructed and $P$ the vector of the measurement values, this linear model can be expressed by [21]:

$$
P=R J
$$

where R, called the Radon matrix, models this linear dependence. Due to the nature of the phenomenon involved in the CT process, $J$ is in fact the absorption map and P the logarithm of the attenuation ratio between the intensity of the incident wave and the intensity measured by the detector [9]. This is due to the fact that the wave decreases in intensity exponentially as it passes through the material of the object. The matrix $\mathrm{R}$ cannot be generally inverted due to its large dimension and the fact that, because the problem is ill-posed, $\mathrm{R}$ is ill-conditionned [14].

The Filtered Back Projection (FBP) algorithm [8], elegantly exploits the fact that $J^{\prime}=$ $R^{T} P=R^{T} R J$, where ${ }^{T}$ is the transposition. $J^{\prime}$ is a smoothed version of $J$ whose smoothing is known in the frequency domain. The method consists of high-pass filtering the measurement vector $\mathrm{P}$ and reconstructing the absorption coefficient map by using the dual operator $R^{T}$ called the back-projection operator. Despite its good formulation, this methods is barely used due to the fact that the high-pass filter, that counteracts the blurring, has no expression in the spatial domain. The need of interpolation in the frequency domain leads to reconstruction artifacts.

Another elegant mathematical solution, based on the Fourier slice theorem [11], consists of using the relationship between the Fourier transform of both the image to be reconstructed and the set of projection measurements. However, as with the FBP, this method needs interpolation in the frequency domain, which also leads to artifacted reconstructed images.

Today, the most used techniques are based on iterative estimation of a solution $\hat{J}$ of Eq. (1) in an optimisation scheme. There are mainly two families of iterative techniques. The first one aims to find the solution $\hat{J}$ that minimizes a square criterion $\|P-R J\|_{2}$ i.e. the $L_{2}$ norm of the projected reconstruction error. We call it the ART family (ART, SIRT, . . .) following the work of Kaczmarz [10]. These techniques are based on assuming that the noise measurement is Gaussian [7], and their convergence is rather slow. The second family is based on assuming that the noise measurement is Poisson distributed. We call it the EM family (MLEM, OSEM, ... ) [18]. They converge faster than those of the ART family, but their values are constrained to be positive [23] [19]. 
2.2 Review on $\mathrm{THz}$ tomography

Applying any of the previously described methods to perform a THz tomography is challenging since they all require a linear model of the measurement process. Such a linear model is almost impossible since the interaction between the $\mathrm{THz}$ wave and the material to be inspected usually involves refraction and reflection. However, current attempt to perform $\mathrm{THz}$ tomography is mainly based on using conventional reconstruction methods.

To our knowledge, there are two main modalities to perform $\mathrm{THz}$ tomography in current literature. The main difference between these two modalities is whether both refraction and reflection losses are considered or not.

The first modality is suitable for reconstructing objects whose shape and material induce little refraction and reflection. The more refraction and reflection loss, the more artifacted the reconstructed attenuation image is.

For example in [15] Recur et al. consider using FBP, SART - Simultaneous Algebraic Reconstruction Technique - and OSEM - Ordered Subset Expectation Maximization - algorithms to reconstruct the absorption coefficient map of the object under inspection. The measurements have been obtained by using a continuous $\mathrm{THz}$ beam. The Radon matrix is designed by considering a Gaussian intensity profile for the $\mathrm{THz}$ beam when propagating through the air.

In the same way, Ferguson et al. [5] propose to directly reconstruct the complex refractive index of the object under inspection, without considering any prior information about this object. To achieve such a reconstruction, they use a pulsed $\mathrm{THz}$ source. They propose a new model of the interaction between this pulse mode wave and the object to interpolate the spectral information in the Fourier domain in a slice Fourier like scheme. The inverse Fourier transformation of the obtained spectral information allows the reconstruction of the object.As a drawback, this method only works with low-refractive-index simple objects with component parts that are large relative to the wavelength of the $\mathrm{THz}$ radiation.

The second modality has to be used when the shape or material of the object under inspection induces refraction or reflection. However, contrarily to the previous modality, since a conventional reconstruction method is used, prior information on the object will be necessary to achieve reconstruction.

For example, Mukherjee et al. [13] consider reconstructing a homogeneous cylinder with a known shape and material. They use this prior knowledge to compensate for the reflection and refraction in the acquired projections. After compensation, the object can be reconstructed by using an FBP algorithm. This method is very suitable for NDT, since it allows the reconstruction of an object that deviates from a known model. However, this method has a drawback [13], it can only be used to reconstruct a defect located in the central part of the cylinder. Moreover, as stated by Tepe et al. [22], such a method is completely dedicated to reconstructing a cylinder and cannot be applied to objects with other shapes. 


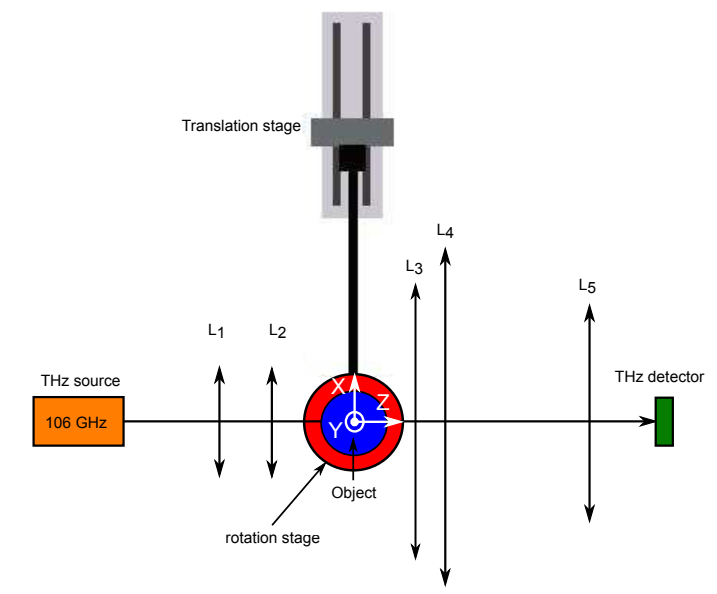

Fig. 1 Experimental THz setup to aquire projections of an object: a THz beam emitted from a THz source is shaped through $L_{1}$ and $L_{2}$ lenses to be focused in the object and then is shaped through $L_{3}, L_{4}$ and $L_{5}$ lenses to be focused on a THz detector. All lenses are planoconvex. Translation and rotation stages are used to move and rotate the object.

\section{Modeling of THz radiation}

A key feature in tomography is the ability to represent how the wave propagates and how it interacts with the objects to be inspected. This model is usually referred to as the projection.

Projection operators have to be of low computational complexity, since they can be potentially used many times when using an iterative reconstruction algorithm. This prohibits projection methods based on finite elements. One of the main ways to simplify the computation of interaction between the beam and the object is to decompose the beam into a discrete set of rays. However, this simplification can lead to a model that is far away from the way the $\mathrm{THz}$ beam propagates and therefore could potentially bias the reconstruction.

In this section we propose to review how this problem has been addressed in the relevant literature and to propose a new model that is likely to achieve a good compromise between complexity and reliability. Firstly, we present our experimental setup. This setup has been used to measure the THz beam intensities after propagating through an object under inspection. It has also been used to acquire the intensity of a $\mathrm{THz}$ beam propagating through the air. Secondly, we propose to show how the model we present is able to simulate the intensity of a $106 \mathrm{GHz} \mathrm{THz}$ beam propagating through the air. Thirdly, we propose to highlights its ability to simulate the attenuation of the same $\mathrm{THz}$ beam propagating through a cylinder with known optical parameters (index of refraction and absorption coefficient).

\subsection{Experimental setup}

The experimental setup we used is plot in Fig. 1. It aims to measure the attenuation the $\mathrm{THz}$ beam undergoes when it propagates through an object placed at the focal point of the setup. The $\mathrm{Z}$ axis corresponds to the optical axis while the $\mathrm{XY}$ plane is perpendicular to 


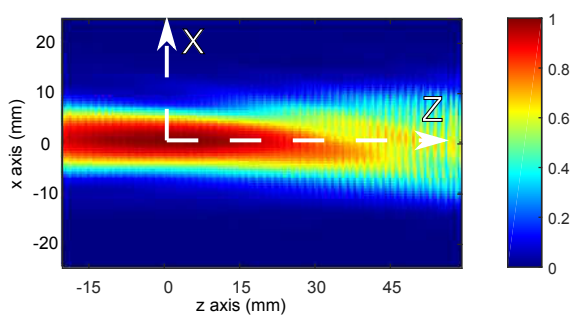

Fig. 2 Acquired intensity map of $\mathrm{THz}$ beam at $106 \mathrm{GHz}$ in the $\mathrm{XZ}$ plane. Colorbars represent intensities of the $\mathrm{THz}$ beam. Intensities are normalized with respect to the maximum intensity of the $\mathrm{THz}$ beam.

the optical axis. We consider a $100 \mathrm{~mW}$ compact $\mathrm{THz}$ source (AB millimetre company) which emits at $106 \mathrm{GHz}$ and corresponds to a $2.82 \mathrm{~mm}$ wavelength in the air. The source frequency was generated through a commercial radio frequency synthesizer (AnaPico company) with a $100 \mathrm{MHz}$ frequency excursion. All lenses are planoconvex. The beam was first collimated through an $L_{1}$ lens and then focused on the object through an L2 lens. $L_{1}$ and $L_{2}$ lenses, made of PolymethylPentene, have a diameter of $50 \mathrm{~mm}$ and focal lengths are 150 and $100 \mathrm{~mm}$ respectively. After propagating through the object, the beam was collected and collimated through $L_{3}$ and $L_{4}$ lenses and finally focused on the detector through lens $L_{5}$. The $L_{3}, L_{4}$ and $L_{5}$ lenses, made of polyethylene, have a diameter of $140 \mathrm{~mm}, 160 \mathrm{~mm}$ and $100 \mathrm{~mm}$ respectively. Their focal lengths are $185 \mathrm{~mm}, 240 \mathrm{~mm}$ and $100 \mathrm{~mm}$ respectively. The T-Waves Technologies detector is based on a High Electron Mobility transistor whose responsivity is about $40 \mathrm{kV} / \mathrm{W}$ at $0.3 \mathrm{THz}$, NEP (noise equivalent power) of $50 \mathrm{pW} / \mathrm{Hz}$ (as formulated in [17]).

By moving and rotating the object, projections are acquired all around the object. The vector of projections $P$ is obtained by considering projections acquired at the same position on the $\mathrm{Y}$ axis.

This experimental setup has also been used to acquire an intensity map of a $\mathrm{THz}$ beam propagating through the air. Such acquisition is mandatory to validate the ability of a given model to simulate the intensity distribution of a $\mathrm{THz}$ beam. This map has been obtained by removing the $L_{3}, L_{4}$ and $L_{5}$ lenses of the experimental setup, and then measuring the beam intensity in different positions of the XZ plane by moving the detector. For this experiment, we measured the $\mathrm{THz}$ beam by moving the detector with a displacement step of $0.5 \mathrm{~mm}$ in both directions. Fig. 2 presents this map where the intensities have been normalized with respect to the maximum intensity value of this $\mathrm{THz}$ beam.

\subsection{Modeling the $\mathrm{THz}$ radiations}

Depending on the refractive index, both reflection and refraction losses may not be the dominant effects. We consider, like Recur et al. [15], that the intensity $I(x, y, z)$ at a location $(x, y, z)$ of a THz beam, when propagated through the air, can be appropriately modeled by a Gaussian equation as follows:

$$
I(x, y, z)=I_{0}\left(\frac{w_{0}}{w(z)}\right)^{2} \exp \left(\frac{-2 r^{2}}{w^{2}(z)}\right)
$$



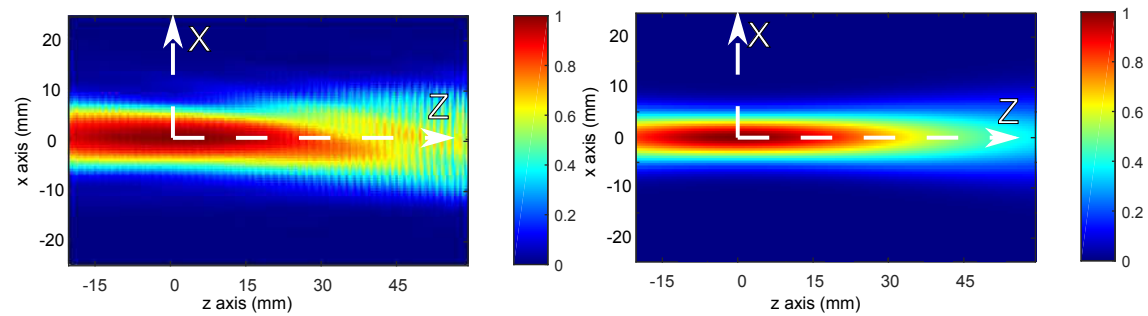

Fig. 3 Intensity map of acquired a) and simulated using the Gaussian modeling (Eq. (2)) b) THz beam at $106 \mathrm{GHz}$ in the $\mathrm{XZ}$ plane. Colorbars represent intensities of the $\mathrm{THz}$ beam. Intensities are normalized with respect to the maximum intensity of the $\mathrm{THz}$ beam.

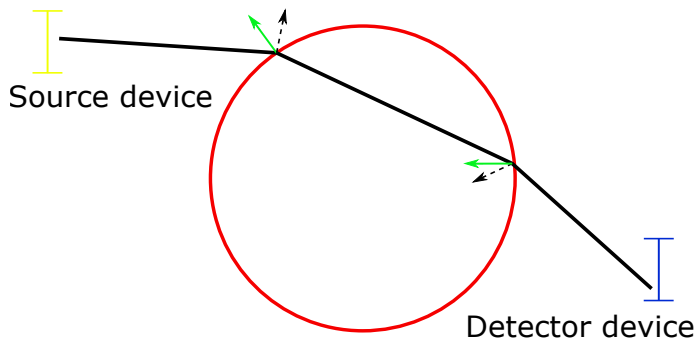

Fig. 4 Snell-Descartes based optical path

where: $\mathrm{r}$ is the distance to the beam axis, $w(z)=w_{0} \sqrt{1+\left(\frac{z}{z_{r}}\right)^{2}}$ the radius of the beam, $w_{0}$, the waist, is the minimum value of the radius, $z_{r}=\frac{\pi w_{0}^{2}}{\lambda}$ is the Rayleigh range, $\lambda$ is the wavelength and $I_{0}$ is the highest intensity value.

Fig.3.b plots a simulation of the acquired intensity map of the $\mathrm{THz}$ beam (see Fig.3.a) by using the Gaussian beam modeling (Eq.(2)). Simulated and acquired intensity maps are similar. However this modeling cannot be used to make a projection operator since it does not consider interaction between $\mathrm{THz}$ beam and an object to be inspected. We depict a simulation of the measurement reported in Fig.2 based on Eq.(2). This shows that this model is relevant for the kind of waves we consider.

A first idea would be to model the $\mathrm{THz}$ beam propagation using a standard Snell \& Descartes ray-tracing (as in X-ray CT). Since Tepe et al. [22] say they neglect the Gaussian beam profile, we presume they used this approach. It has the advantage of easily modeling refraction, reflection and absorption. The global procedure is illustrated in Fig.4: the beam is refracted at each interface and attenuated as it propagates through an object whose absorption index is known. As aknowledged by Tepe et al. [22], this model cannot achieve a reliable projection operation since the intensity profile of the $\mathrm{THz}$ beam is ignored.

Mukherjee et al. [13], propose to account for both reflection and refraction losses by regularly discretizing the $\mathrm{THz}$ pulse in several rays. Each ray has an intensity proportional to its angular distance to the optical axis, following a normal distribution. Each ray is processed by applying the usual ray tracing method for estimating the intensity it carries to the detector, 


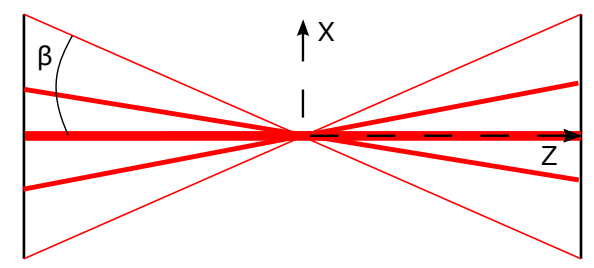

Fig. 5 FRT modeling: the THz beam is regularly discretized in several rays, all intersecting at the position $(0,0,0)$. In this illustration, the width of a ray is connected to its intensity. The wider a ray, the higher its intensity is. The numerical aperture $\beta$ corresponds to the orientation of rays with highest angular distance to the optical axis.
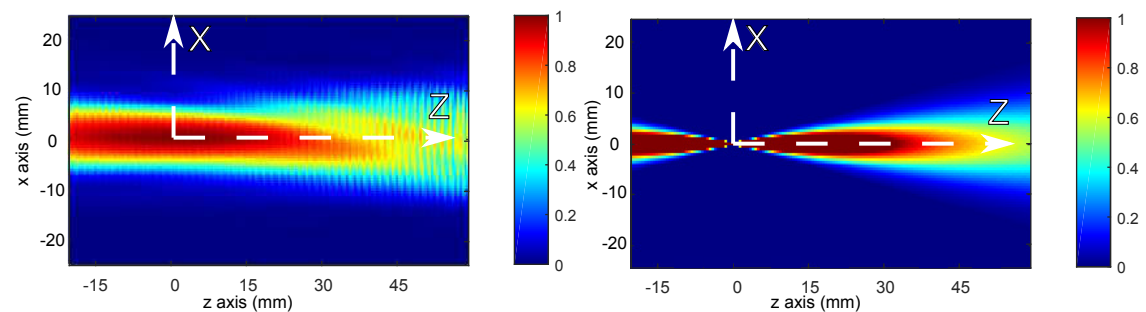

Fig. 6 Intensity map of THz beam at $106 \mathrm{GHz}$ in the XZ plane: acquired a) simulated using the FRT modeling b). Colorbars represent intensities of the $\mathrm{THz}$ beam. Intensities are normalized with respect to the maximum intensity of the THz beam.

if the ray reaches the detector. We call this kind of procedure Fan Ray Tracing (FRT). It is illustrated in Fig.5.

Fig.6.b plots a simulation of THz beam (plotted in Fig.6.a) by using an FRT approach using 100,000 rays. We observe that FRT induces a point-like focalisation, which highly differs from the measured beam shape. As the Rayleigh range contains most of the intensity, such a focalisation results in different beam attenuation compared to a measured beam. Such a deviation is likely to produce differences between acquired and simulated measurements. Knowing this, Mukherjee et al. propose to simulate measurements at the edge of the object by using a Gaussian equation. Although this solution improves simulated measurement at the edge of the object, it does not counteract the point-like focalisation near the center of the object. As a last potential drawback, the systematic sampling of the beam makes the resulting approximation highly dependent on the sample step.

\subsection{Monte Carlo slash ray tracing}

We propose a projection operator that better mimics how the $\mathrm{THz}$ beam propagates through the air. This operator is likely to perform better than FRT to simulate how the THz beam interacts with an object. This projection operator, called Monte Carlo Slash Ray Tracing (MCSRT), is based on a random selection of rays complying with the distribution modeled by Eq.(2).

Let us consider the parameters of Eq.(2). Within our approach, each ray is defined by two randomly selected parameters $\varphi$ and $\psi$, as depicted in Fig.7. We represent in green the 


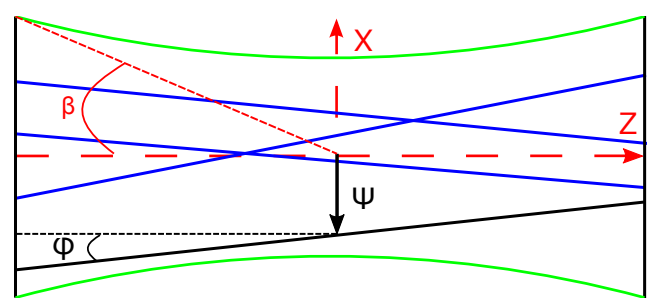

Fig. 7 MCSRT modeling: due to the random selection of parameters $\varphi$ and $\psi$, the rays do not intersect at the position $(0,0,0)$. Each ray have the same intensity

edges of the $\mathrm{THz}$ beam with a numerical aperture $\beta$. The rays launched with the MCSRT modeling are represented in blue and black. $\varphi$ (respectively $\psi$ ) is randomly selected by

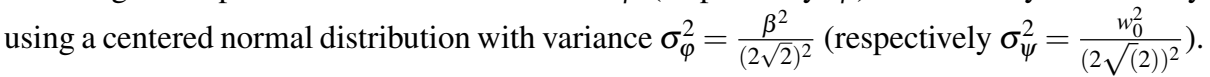
Variance values are obtained by identifying Eq.(2) with the MC approach. The bigger $\sigma_{\varphi}^{2}$ (respectively $\sigma_{\psi}^{2}$ ), the wider the numerical aperture (respectively the beam waist) of the simulated beam is. As illustrated in Fig.7, all rays do not intersect at the center of the beam as within the FRT modeling.

Fig.8.b plots a simulation of the intensity map of the THz beam plotted in Fig.8.a by using the MCSRT model with 100,000 rays. It seems obvious that the proposed model better simulates the energy distribution of a $\mathrm{THz}$ beam propagating through the air than the FRT model, especially in the Rayleigh range.

To confirm this subjective result, we propose to use the 3 error criteria that are intensively used in the signal processing domain: the mean square error, the mean absolute error and the maximum absolute error. The smaller those criteria, the better the simulation is. These criteria are computed as follow:

$$
\begin{aligned}
& \text { Mean square error }=\frac{1}{n} \sum_{i=1}^{n}\left(Q_{i}-\hat{Q}_{i}\right)^{2}, \\
& \text { Mean absolute error }=\frac{1}{n} \sum_{i=1}^{n}\left|Q_{i}-\hat{Q}_{i}\right|, \\
& \text { Max absolute error }=\max _{i=1}^{n}\left(\left|Q_{i}-\hat{Q}_{i}\right|\right),
\end{aligned}
$$

where $Q_{i}$ (respectively $\hat{Q}_{i}$ ) is the $i^{\text {th }}$ pixel value in the intensity map of acquired (respectively simulated) THz beam and $n$ is the total number of pixel in the map. Table1 reports the comparison between the acquired intensity map and its simulation by using the Gaussian beam, FRT and MCSRT modeling (Eq. (3), (4) and (5)). MCSRT modeling clearly outperforms FRT modeling in every criteria. Its error values are comparable to those obtained by using simulations based on the Gaussian beam model (Eq. (2)).

\subsection{Simulated projections comparison}

In the previous section, we compared the ability of both FRT and MCSRT models to simulate how the $\mathrm{THz}$ beam propagates through the air. In this section we propose to compare 

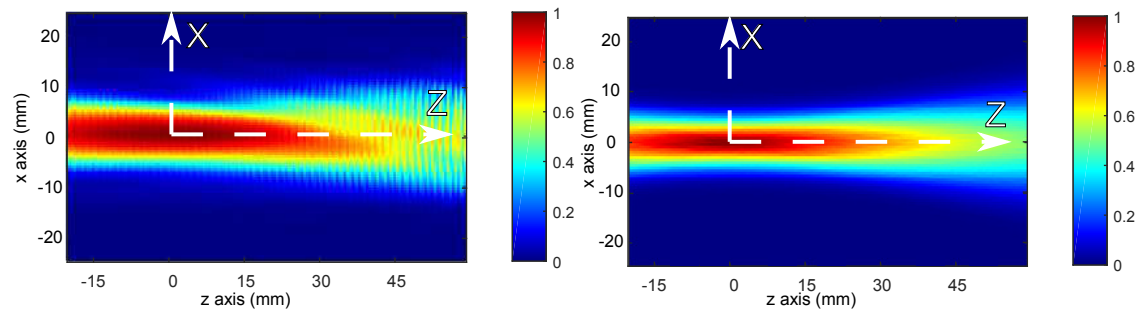

Fig. 8 Intensity map of THz beam at $106 \mathrm{GHz}$ in the XZ plane: acquired a) and simulated using the MCSRT modeling $\mathrm{b}$ ). Colorbars represent intensities of the $\mathrm{THz}$ beam. Intensities are normalized with respect to the maximum intensity of the $\mathrm{THz}$ beam.

Table 1 Comparison between acquired and simulated intensity map of THz beam propagating through the air at $106 \mathrm{GHz}$ using Gaussian beam (Eq. (2)), FRT and MCSRT modeling.

\begin{tabular}{lclc}
\hline & Mean square error (Eq. (3)) & Mean absolute error (Eq. (4)) & Max absolute error (Eq. (5)) \\
\hline Gaussian beam (Eq. (2)) & 0.02 & 0.08 & 0.39 \\
FRT & 0.04 & 0.11 & 1.00 \\
MCSRT & 0.01 & 0.06 & 0.38 \\
\hline
\end{tabular}

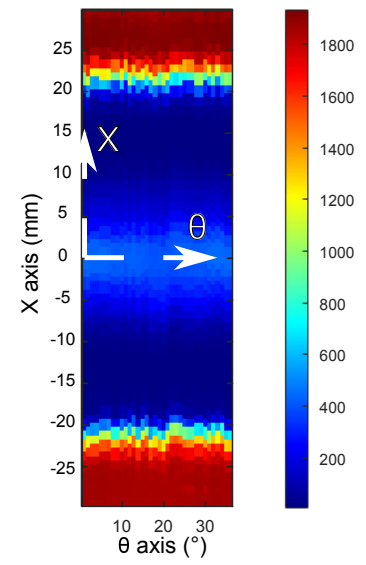

Fig. 9 Acquired sinogram of the first object. Colorbar indicates acquired projections $(\mathrm{mV})$ all around the first object. The angular step sampling is about $10^{\circ}$.

the ability of both methods to simulate a projection measurement. To achieve this comparison, we acquired projections of a $40 \mathrm{~mm}$ diameter Polyoxymethylene (POM) cylinder at $106 \mathrm{GHz}$ (see Fig. 9). Refractive index (respectively absorption coefficient) is about 1.7 (respectively $0.35 \mathrm{~cm}^{-1}$ ) at $106 \mathrm{GHz}$. Acquired and simulated projections, at a specific $\theta$, for both methods are depicted in Fig.10. Both acquired and simulated projections are normalized with regard to its maximum projection value (i.e. when $\mathrm{THz}$ beam propagate through the air). Simulated projections using FRT and MCSRT modeling are obtained by using 100,000 rays. We can see that MCSRT projections values at the edge of the cylinder gradually increase. Using the FRT method, the transition between the cylinder and the air is abrupt. This is likely to be caused by the fact that the FRT method does not suitably model the beam in the focusing range. This is more obvious in Fig. 11 where absolute differ- 


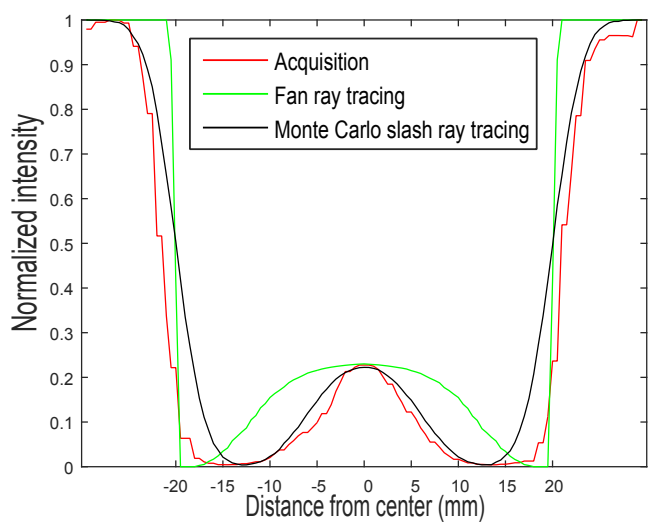

Fig. 10 Simulated projections of a homogeneous cylinder $\left(n=1.7, \alpha=0.35 \mathrm{~cm}^{-1}\right.$ at $\left.106 \mathrm{GHz}\right)$ using FRT (green) and MCSRT (black) modeling compared with acquired projections (red)

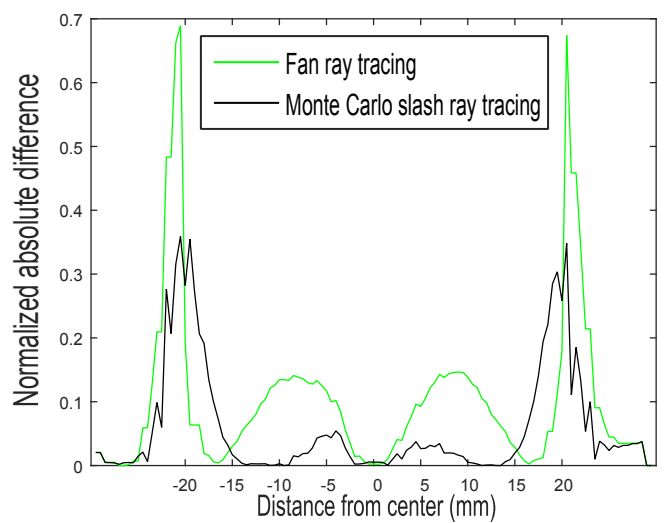

Fig. 11 Normalized absolute error between acquired and simulated projections of a homogeneous cylinder $\left(n=1.7, \alpha=0.35 \mathrm{~cm}^{-1}\right.$ at $106 \mathrm{GHz}$ ) using FRT (green) and MCSRT (black) modeling

Table 2 Comparison between acquired and simulated projections with a THz beam propagating through a cylinder at $106 \mathrm{GHz}$ using FRT and MCSRT modeling.

\begin{tabular}{lclc}
\hline & Mean square error (Eq. (3)) & Mean absolute error (Eq. (4)) & Max absolute error (Eq. (5)) \\
\hline FRT & 0.02 & 0.09 & 0.67 \\
MCSRT & 0.01 & 0.06 & 0.36 \\
\hline
\end{tabular}

ences between acquired and simulated projections are depicted. MCSRT leads to simulated projections that are closer to acquired projections than simulated projections given by FRT.

To confirm this subjective result, we used the same error criteria as in section 3.3, where this time $Q_{i}$ (respectively $\hat{Q}_{i}$ ) correspond to the $i^{\text {th }}$ acquired (respectively simulated) projection and $\mathrm{n}$ is the total number of projections. Results reported in Table 2 show that every criterion is smaller when considering the MCSRT model than when considering the FRT model. Thus the MCSRT method outperforms the FRT method for simulating the projection measurements. 


\section{From object reconstruction to defect reconstruction}

The method we propose is similar to [15] in that it uses a conventional reconstruction technique. But, where Recur et al. reconstruct an unknown object with low refraction and reflection, we propose to consider objects that have reflection and refraction, and to reconstruct the deviation between the object to be inspected and its known CAD model. This technique is completely relevant in a NDT context.

Let $P$ be the measurement and $J$ be the image of absorption coefficient to be reconstructed. The non-linear projection model can be written as:

$$
P=\mathscr{F}(J) \text {. }
$$

Let $J_{0}$ be the CAD based absorption coefficient of the object to be reconstructed. Let us denote $\Delta J$ the deviation between $J$ and $J_{0}$ :

$$
J=J_{0}+\Delta J .
$$

Based on (7), a first order series expansion of Eq.(6) gives:

$$
P=\mathscr{F}\left(J_{0}\right)+\frac{\partial \mathscr{F}}{\partial J}\left(J_{0}\right) \Delta J+O^{2}
$$

where $\mathscr{F}\left(J_{0}\right)$ is obtained by simulating the measurement using a CAD model of the object, $\frac{\partial \mathscr{F}}{\partial J}\left(J_{0}\right)$ is the Jacobian matrix of $\mathscr{F}$ around $J_{0}$ and $O^{2}$ is a second order term. By discarding second order terms, Eq. (8) can be rewritten as a linear equation:

$$
\Delta P=R \Delta J,
$$

where $\Delta P=P-\mathscr{F}\left(J_{0}\right)$ is the deviation between the measurements and the simulated measurements based on the CAD model, and $R=\frac{\partial \mathscr{F}}{\partial J}\left(J_{0}\right)$ is a Radon-like matrix whose coefficients can be computed in the same way as [15] but taking into consideration the model with reflection and refraction losses we proposed in section 3 .

The reconstruction we propose is based on the SART algorithm [1]:

$$
\Delta J^{k+1}=\Delta J^{k}+\rho R^{T}\left(\Delta P-R \Delta J^{k}\right),
$$

where $R$ and $R^{T}$ are respectively the projection and back-projection matrices linking the reconstructed pixel $\Delta J^{k}$ at iteration $k$ to $\Delta P, \rho$ is a relaxation parameter that ensures the convergence of the algorithm [4] and $\Delta J^{0}$ is an initial value that can be set to zero.

The choice of an ART-like algorithm is motivated by the fact that $\Delta J$ can have positive and negative values. An algorithm of the EM family would only have reconstructed positive values (see Section 2).

Now, noise in the projection can lead to a divergence of the iterative reconstruction algorithm (overfitting). Since no prior information on the defect to be reconstructed is available, adding a regularization term in the reconstruction process is rather risky. Therefore, we propose to regularize by stopping the iterative reconstruction process before convergence (early stopping) [6].

Finally, the previously proposed method can be modified by considering replacing the simulated projections $\mathscr{F}\left(J_{0}\right)$ in Eq.(8) by acquired projections using an object know to have 
no defect. Such a modification could have relevant positive effects on the reconstruction process since the measured projection could include optical effects that are not included in the CAD-based projection model. With this modification, $\Delta P$ only accounts for deviations induced by defects, but could potentially double the effect of measurement noise.

\section{Experiments}

In this section, we present a series of experiments that illustrates both performances and limitations of our method. A preliminary remark is necessary to better understand the following experiments. Due to the difference between the refractive index of air and the refractive index of material composing the object under inspection, a blind zone appears where, depending on the relative orientation of the beam and the object, the incoming energy may not reach the detector. Therefore, if the defect is located in this blind zone, there can be some incoherence between measured and expected projections, since some information is missing. Thus, the quality of the reconstructed defect may depend on the relative location of the defect in the object [13].

The four experiments we propose consist of reconstructing four different cylindrical shaped objects with different defect locations. Cylindrical shaped objects such as particle filters are common in an NDT context. The acquisition has been performed by acquiring projections of a $106 \mathrm{GHz} \mathrm{THz}$ beam with an angular step of $10^{\circ}$ and an $\mathrm{x}$ displacement step of $0.5 \mathrm{~mm}$ for each of the four objects.

As in [22] and in [13], we consider materials with a high refractive index. The reconstruction algorithm is stopped after 50 iterations since, during all the experiments we carried out, it seemed to be a good trade off between artefacts and convergence. All the plots are presented in a $[-0.50,0.50] \mathrm{cm}^{-1}$ range to ease the comparison between experimental results. All defects are also cylindrical shaped. Their size after reconstruction is measured by their full width at half maximum.

\subsection{Cylinder with no defect}

The first object is the POM cylinder presented in Section 3 with no known deviation from its CAD model. The refractive index of POM at $106 \mathrm{GHz}$ is 1.7 , while its absorption coefficient is $0.35 \mathrm{~cm}^{-1}$. Fig. 12.a depicts a visible photography of this first object.

Fig.12.b depicts the reconstructed deviation obtained after 50 iterations of the reconstruction algorithm. As expected, the reconstructed attenuation is only composed of distributed artifacts whose values range from $[-0.01,0.01] \mathrm{cm}^{-1}$ with a mean value close to $0.00 \mathrm{~cm}^{-1}$. Fig. 12.c depicts horizontal profile at the center of the first object: all values are close to 0 . This tends to validate our projection method.

\subsection{Cylinder with a central defect}

The second object is a $100.0 \mathrm{~mm}$ diameter PolyCarbonate (PC) cylinder with a $20.0 \mathrm{~mm}$ diameter Acrylonitrile Butadiene Styrene (ABS) cylindrical central inclusion. Optical pa- 


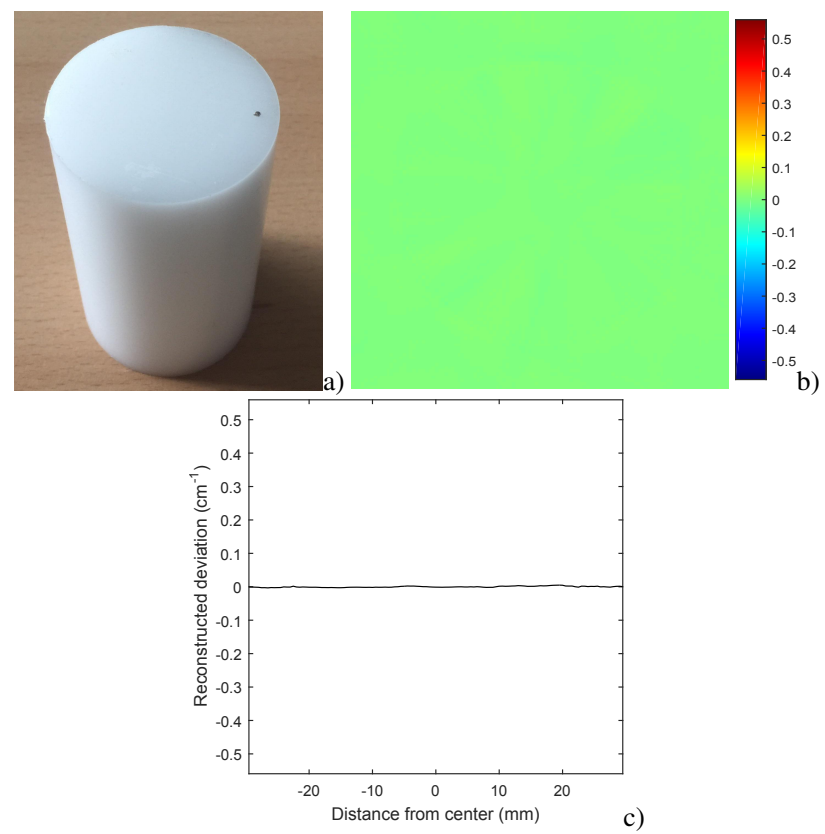

Fig. 12 First object, cylinder with no defect: visible photography a), reconstructed deviation $\left.\left(\mathrm{cm}^{-1}\right) \mathrm{b}\right)$ and horizontal plot profile centered on the first object c).

rameters of both $\mathrm{PC}$ and $\mathrm{ABS}$ are known at $106 \mathrm{GHz}$ : respectively the refractive indexes are 1.6 and 1.5 while the absorption coefficients are respectively $0.25 \mathrm{~cm}^{-1}$ and $0.55 \mathrm{~cm}^{-1}$. Fig.13.a depicts a visible photography of the second object. A red dashed circle surrounds the defect.

Fig.13.b depicts the reconstructed deviation obtained after 50 iterations of the reconstruction algorithm. The reconstructed defect is a cylinder located at the center whose diameter is close to $20.5 \mathrm{~mm}$. As expected, the reconstructed absorption deviation in the central part of the image is close to $0.30 \mathrm{~cm}^{-1}$.

Obviously, the reconstructed image is more artifacted than in the previous experiment. These artifacts are mainly induced by optical effects that are not taken into account since the model is based on a PC cylinder without defects. Stopping the reconstruction algorithm earlier could reduce these effects, as illustrated in Fig.14.a. However in this case the central value does not reach the expected deviation value of $0.30 \mathrm{~cm}^{-1}$.

Fig.14.b depicts the horizontal central profile of Fig.13.b and Fig.14.a. We can observe that, after only 10 iterations, the defect shape and location is already identifiable. Only the values are wrong. Therefore, for NDT, considering earlier stopping can be envisaged to prevent the upcoming of reconstruction artifacts. Moreover, we can decide whether or not an object deviates from its CAD model by thresholding the reconstructed image. The threshold could have been determined with a set of objects whose manufactured quality is known to match the specifications. 

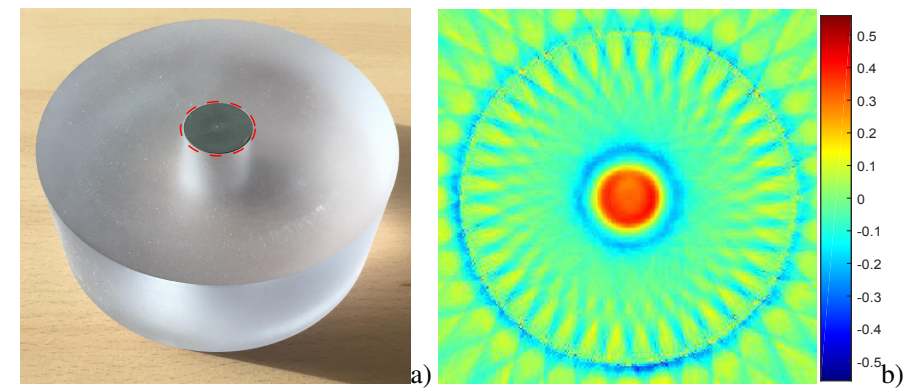

Fig. 13 Second object, cylinder with a central defect: visible photography a), reconstructed deviation $\left(\mathrm{cm}^{-1}\right)$ b).
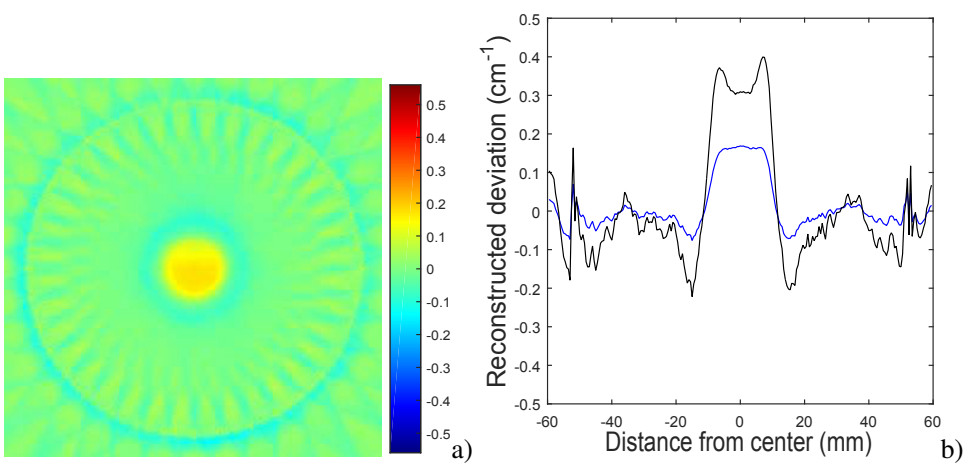

Fig. 14 Reconstructed deviation of the second object after 10 iterations $\left(\mathrm{cm}^{-1}\right)$ a) and horizontal plot profile of reconstructed deviation of the second object after 10 (blue) and 50 (black) iterations b).

\subsection{Cylinder with a defect close to the center}

The third object is a $40.0 \mathrm{~mm}$ POM cylinder with a $5.0 \mathrm{~mm}$ diameter cylindrical hole (filled with air) located at $1.0 \mathrm{~mm}$ from the center. The optical parameters of this POM cylinder are different from those of the first cylinder since the supplier is different. Its refractive index at $106 \mathrm{GHz}$ is 1.7 and its absorption coefficient is $0.55 \mathrm{~cm}^{-1}$. We consider the refractive index of the air to be 1.0 with no absorption (absorption coefficient is $0.00 \mathrm{~cm}^{-1}$ ). Since air and POM have very different refractive indexes, the air cylinder will induce refraction. Fig.15.a depicts a visible photography of the third object with a red dashed circle surrounding the defect.

Fig.15.b depicts the reconstructed deviation obtained after 50 iterations of the reconstruction algorithm. The diameter of the reconstructed defect is close to $7.8 \mathrm{~mm}$ and its center is located at $3.1 \mathrm{~mm}$ from the center of the POM cylinder. The mean value of the reconstructed absorption coefficient deviation in the air cylinder is close to $-0.42 \mathrm{~cm}^{-1}$ which differs from the expected value of $-0.55 \mathrm{~cm}^{-1}$. This is also true for its diameter. This situation can easily be explained by the partial volume effect induced by the fact that the radius of the air cylinder is smaller than the waist. 


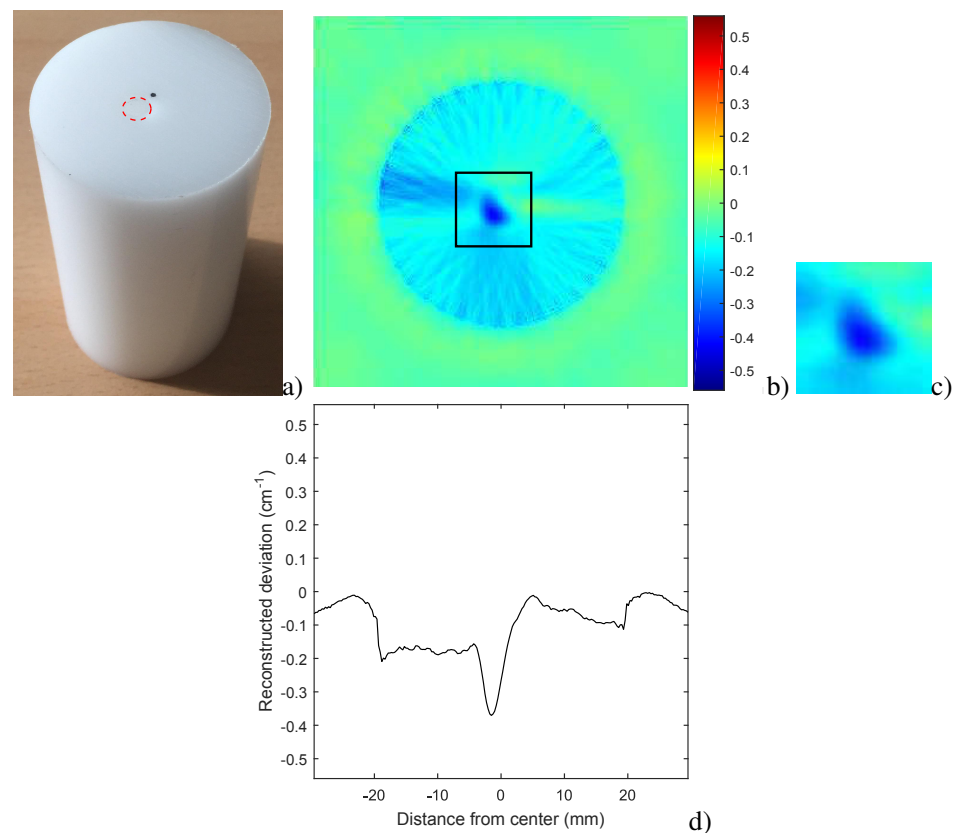

Fig. 15 Third object, cylinder with a defect near the center: visible photography a), reconstructed deviation $\left.\left(\mathrm{cm}^{-1}\right) \mathrm{b}\right)$ with zoom on the defect c) and horizontal plot profile centered on the defect d).

One can observe that, in addition to not having the right dimension, the reconstructed air cylinder also has the wrong shape (see Fig.15.c). This can be explained by the fact that the model we use does not consider all optical effects (e.g. diffraction due to the air/POM interfaces at the edges of the cylinder).

Fig.15.d depicts the horizontal plot profile centered on the defect. A dissymmetry in the artifacts is clearly visible. This dissymmetry may be induced by the non central position of the defect in the object. In fact, this dissymmetry is in the same direction as the deviation of the defect from the center.

To account for additional optical effects induced by the POM cylinder, we propose to replace $\mathscr{F}\left(J_{0}\right)$ in Eq.(8) by acquired projections of the same object, but with no defect (as mentioned in Section 4). The result of this modification is presented in Fig.16.a.

It can be seen that the shape, size and position of the reconstructed defect is closer from its expected shape, size and position (see Fig.16.b): the location of the center is close to $1.0 \mathrm{~mm}$ while its diameter is about $4.8 \mathrm{~mm}$. Fig.16.c depicts the horizontal plot profile centered on the defect. Replacing the simulation by a real measurement for computing $\Delta P$ in Eq. (10) allows to consider optical phenomena induced by the POM cylinder which are not considered by the MCSRT modeling. This experiment suggests that this replacement is relevant in an NDT context. 

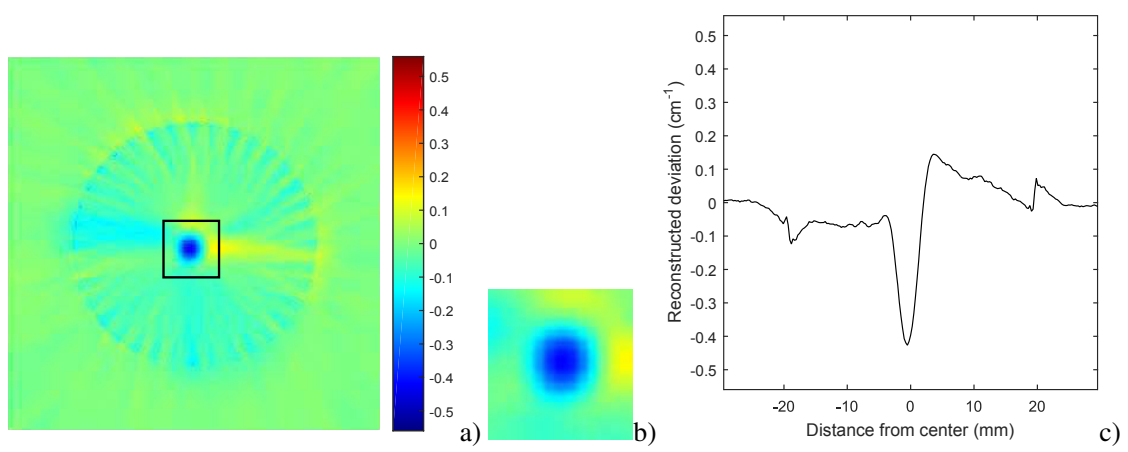

Fig. 16 Reconstructed deviation of the third object with the defect located at $1.0 \mathrm{~mm}$ from the center using acquired measurement $\left(\mathrm{cm}^{-1}\right)$ a) with zoom on the defect b) and horizontal plot profile centered on the defect c).

5.4 Cylinder with defect near the periphery

The fourth object is also a $40.0 \mathrm{~mm}$ POM cylinder with a $5.0 \mathrm{~mm}$ diameter cylindrical hole. But this time the hole is located at $10.0 \mathrm{~mm}$ from the center, i.e. in the blind zone. Fig.17.a depicts a visible photography of this fourth object with a red dashed circle surrounding the defect.

Fig.17.b depicts the reconstructed deviation obtained after 50 iterations of the reconstruction algorithm. Now the cylindrical shape of the defect is not properly reconstructed. At the location of the defect - which is indicated by a red dashed circle - a pie-shaped form has been reconstructed. Fig17.c depicts the horizontal plot profile centered on the defect. This seems to derive from the lack of information induced by refraction in the blind zone [13]. To corroborate this interpretation, i.e. this does not result from any other phenomena other than refraction, reflection and absorption losses, we have simulated this experiment. The result of this simulation is depicted in Fig. 18.a. Fig18.b depicts the horizontal plot profile centered on the defect. The reconstructed shape and location are identical.

This experiment suggests that reconstructing a defect in the blind zone can be problematic. However, in an NDT context, this method is still relevant to decide whether or not the object has a defect. Moreover, the reconstructed defect is a good marker of the real defect since the real defect is located inside the reconstructed defect.

\section{Discussion}

The third experiment tends to show that using acquired projections instead of simulated projections increases the quality of defect reconstruction. This highlights the fact that optical effects other than those that our model accounts for should be considered. The replacement we proposed is a good method to avoid this problem, but modeling these missing optical effects should be a better solution.

We noted, in the fourth experiment, that the incoherence between projection measurements and model induces imprecisions in the reconstruction. This is mainly due to the fact 


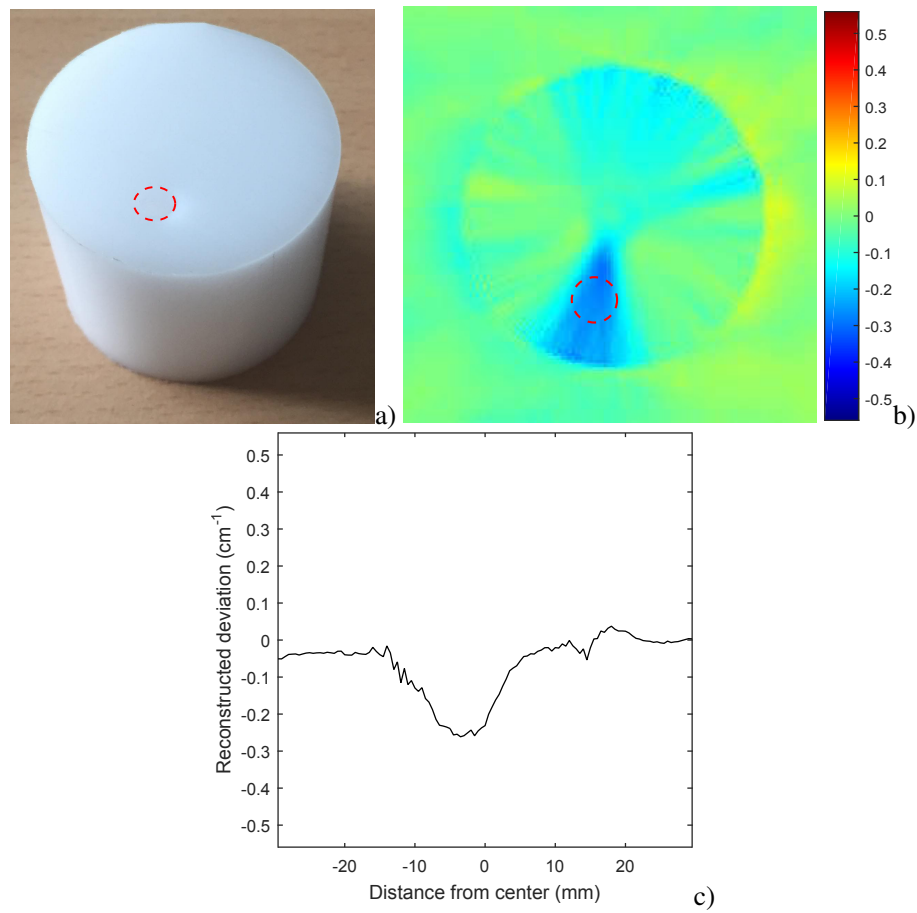

Fig. 17 Fourth object, cylinder with a defect near the periphery: visible photography a), reconstructed deviation $\left.\left(\mathrm{cm}^{-1}\right) \mathrm{b}\right)$ and horizontal plot profile centered on the defect $\left.\mathrm{c}\right)$. A red dashed circle indicate the position of the defect.
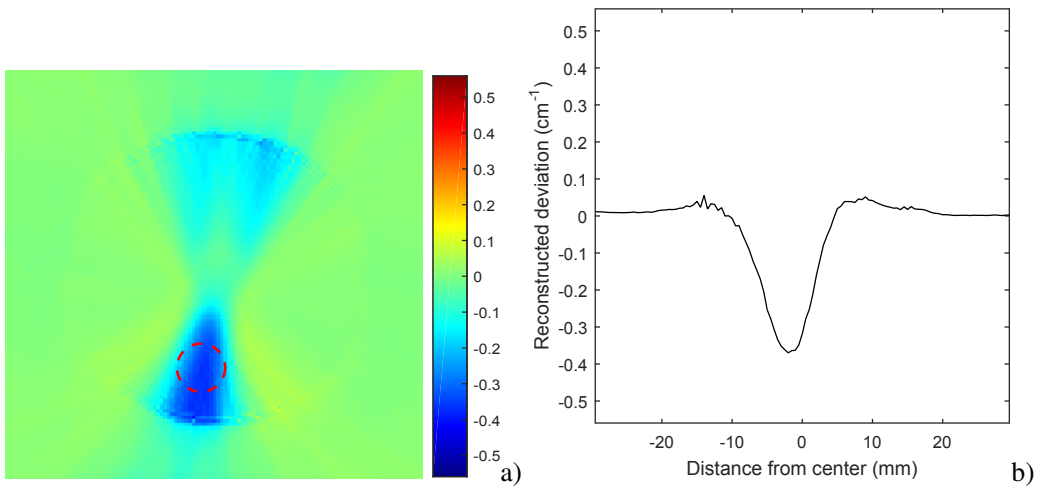

Fig. 18 Reconstructed deviation of the fourth object with the defect located at $10 \mathrm{~mm}$ from the center using synthetic measurement $\left(\mathrm{cm}^{-1}\right)$ a) and horizontal plot profile centered on the defect $\left.\mathrm{b}\right)$.

that, when a defect is placed in what Mukherjee et al. [13] call the blind zone, information is missing since the beam does not reach the detector (partially or completely). Within our model, this missing measurement is seen as a full absorption, when it is in fact refraction. A way to circumvent this problem could be to retrieve lost information by moving the detector in the plane perpendicular to the optical axis, or to use a detector strip (with 2D reconstruction) or a detector matrix (with $3 \mathrm{D}$ reconstruction). 


\section{Conclusion}

In this paper, we have presented a Terahertz (THz) based tomography dedicated to non destructive inspection. The two key features of this contribution are:

- a new model for the interaction between radiation and objects to be inspected,

- a reconstruction algorithm based on linearization about known CAD models.

The interaction model we have proposed is based on a Monte Carlo extension of usual ray tracing methods. It aims to account for the Gaussian intensity profile of $\mathrm{THz}$ beam and considers refraction and reflection losses when propagating through an object. The experiments we carried out show that this model performs better, with a reasonable computation complexity, than other methods currently proposed in relevant literature.

The reconstruction algorithm we have proposed consists of modeling the propagation of the wave through the known CAD model of the object to be inspected with a Radonlike matrix accounting for both reflexion and refraction and to use this projection model to reconstruct the deviation of the object to its known CAD model. If this CAD model does not correspond to the object to be characterized, a difference between measured and simulated projections will be visible. This difference between simulated and measured projections is used to ensure the detection and localization of the defect.

Several directions can be envisaged for future work. First, it could be beneficial to update the projection model during reconstruction. This modification is pivotal, since it would allow reconstruction of major defects inducing high refraction loss. Achieving such a change means being able to simulate the propagation of the $\mathrm{THz}$ beam in a discrete object. It also means proving the convergence of such an iterative reconstruction method. Second, it would be of prime importance to include diffraction to our model. Finally, we envisage modifying the acquisition process by moving the detector device perpendicularly to the beam propagation axis, or by using detector strips or matrices. We expect this additional information will help to overcome the problem of the blind zone. This will naturally induce a more complex model and eventually a more complex reconstruction algorithm.

\section{References}

1. Andersen, A., Kak, A.C.: Simultaneous algebraic reconstruction technique(sart) : A superior implementation of the art algorithm. Ultrasonic imaging 6(1), 81-94 (1984)

2. Bailey, D.L., Townsend, D.W., Valk, P.E., Maisey, M.N.: Positron Emission Tomography: Basic Sciences. Springer Science \& Business Media (2006)

3. Chiesura, G., Luyckx, G., Voet, E., Lammens, N., van Paepegem, W., Degrieck, J., Dierick, M., van Hoorebeke, L., Vanderniepen, P., Sulejmani, S., Sonnenfeld, C., Geernaert, T., Berghmans, F.: A microcomputed tomography technique to study the quality of fibre optics embedded in composite materials. Sensors 15(5), 10852-10871 (2015)

4. Elfving, T., Hansen, P.C., Nikazad, T.: Semiconvergence and relaxation parameters for projected sirt algorithms. SIAM Journal on Scientific Computing 34(4), A2000-A2017 (2012)

5. Ferguson, B., Wang, S., Gray, D., Abbot, D., Zhang, X.C.: T-ray computed tomography. Optics Letters 27(15), 1312-1314 (2002)

6. Flemming, H.: Equivalence of regularization and truncated iteration in the solution of ill-posed image reconstruction problems. Linear Algebra and its Applications 130, 133-150 (1990)

7. Gordon, R., Bender, R., Herman, G.: Algebraic reconstruction techniques (art) for three-dimensional electron microscopy and X-ray photography. Journal of Theoretical Biology 29(3), 471-476 (1970)

8. Guillet, J.P., Recur, B., Frederique, L., Bousquet, B., Canioni, L., Manek-Hnninger, I., Desbarats, P., Mounaix, P.: Review of terahertz tomography techniques. Journal of Infrared, Millimeter, and Terahertz Waves 35(4), 382-411 (2014) 
9. Hsieh, J.: Computed Tomography: Principles, Design, Artifacts, and Recent Advances. SPIE Press (2003)

10. Kaczmarz, S.: Approximate solution of systems of linear equations. International Journal of Control 57(6), 1269-1271 (1993)

11. Kak, A.C., Slaney, M.: Principles of Computerized Tomographic Imaging. Society of Industrial and Applied Mathematics (2001)

12. Mittleman, D., Hunsche, S., Boivin, L., Nuss, M.C.: T-ray tomography. Optics Letters 22(12), 904-906 (1997)

13. Mukherjee, S., Federici, J., Lopes, P., Cabral, M.: Elimination of fresnel reflection boundary effects and beam steering in pulsed terahertz computed tomography. Journal of Infrared, Millimeter, and Terahertz Waves 34(9), 539-555 (2013)

14. Natterer, F.: The Mathematics of Computerized Tomography. Society for Industrial and Applied Mathematics (2001)

15. Recur, B., Guillet, J., Manek-Hönninger, I., Delagnes, J., Benharbone, W., Desbarats, P., Domenger, J.P., Canioni, L., Mounaix, P.: Propagation beam consideration for $3 \mathrm{~d}$ thz computed tomography. Optics Express 20(6), 5817-5829 (2012)

16. Reiser, M.F., Semmler, W., Hricak, H.: Magnetic Resonance Tomography. Springer Science \& Business Media (2007)

17. Schuster, F., Coquillat, D., Videlier, H., Sakowicz, M., Teppe, F., Dussopt, L., Giffard, B., Skotnicki, T., Knap, W.: Broadband terahertz imaging with highly sensitive silicon cmos detectors. Optics express 19(8), 7827-7832 (2011)

18. Shepp, L.A., Vardi, Y.: Maximum likelihood reconstruction for emission tomography. IEEE Transactions on Medical Imaging 1(2), 113 - 122 (1982)

19. Slambrouck, K.V., Stute, S., Comtat, C., Sibomana, M., Velden, F.H.P.V., Boellaard, R., Nuyts, J.: Bias reduction for low-statistics pet: Maximum likelihood reconstruction with a modified poisson distribution. IEEE Transactions on Medical Imaging 34(1), 126 - 136 (1982)

20. Stock, S.: X-ray microtomography of materials. International Materials Reviews 44(4), 141-64 (2015)

21. Strauss, O., Lahrech, A., Rico, A., Mariano-Goulart, D., Telle, B.: Nibart: A new interval based algebraic reconstruction technique for error quantication of emission tomography images. In: MICCAI: Medical Image Computing and Computer-Assisted Intervention. London, United Kingdom (2009)

22. Tepe, J., Schuster, T., Littau, B.: A modified algebraic reconstruction technique taking refraction into account with an application in terahertz tomography. Journal Inverse Problems in Science and Engineering 25(10), 1448-1473 (2016)

23. Yokoi, T., Shinohara, H., Hashimoto, T., Yamamoto, T., Niio, Y.: Implementation and performance evaluation of iterative reconstruction algorithms in spect. In: Second International Workshop on EGS. Tsukuba, Japan (2000) 